\title{
Interpretation of James' Experiments in Plasma Theory of Solar Radar Echoes
}

\author{
V.N. Mel'nik \\ Institute of Radio Astronomy, Krasnoznamyonnaya St.4, 310002, \\ Kharkov, Ukraine
}

\begin{abstract}
In the framework of the plasma theory of solar radar echoes results of the known James' experiments such as the values of effective cross-sections, the anisotropy of reflections, the spectrum of reflected signals, the heights of radar scattering and others are explained. In particular in this theory reflection of radar signal with high effective crosssections $\left(\sigma=(10-100) \pi R_{\odot}^{2}\right)$ occur in processes $t+l \rightleftharpoons t+l$ on the Langmuir turbulance generated by Type III electrons at altitudes $(1.4-1.6) R_{(\odot)}$ when electron streams propagate towards the Earth. If the electrons move away from the Earth then the scattering cross-sections due to process $t+l \rightleftharpoons t+l$ are small $\left(\sigma<\pi R_{(\cdot)}^{2}\right)$. Reflections from the heights up to $5 R_{\odot}$ can occur at scattering of radar wave on the ion-sound turbulance (processes $t+s \rightleftharpoons t$ ) accompanied the Langmuir turbulance.
\end{abstract}

From 1961 to 1969 James (James 1966, 1970) carried out the solar radar experiments at frequency $f_{0}=38.25 \mathrm{MHz}$. He obtained the following results: scattering cross-sections, $\sigma$, changed from day to day and were high (up to $800 \pi R_{\odot}^{2}$ ), moderate $\left((1-2) \pi R_{(\odot)}^{2}\right)$ and low $\left(<\pi R_{\odot}^{2}\right)$; main reflections occurred at heights $(1.3-1.6) R_{\odot}$ though there were reflections from heights $(2-3) R_{\odot}$ and sometimes even up to $5 R_{\odot}$, but with small cross-sections; frequency shifts of echoes were $20-60 \mathrm{kHz}$. James pointed out that there was good correlation between the radio echo data and solar activity specifically the locality of active region on the solar disk.

The standard theory (Kerr 1952; Bass \& Braude 1957) predicts practically steady reflections from the heights where the local plasma frequency equals the radar frequency $\omega_{p e}=\omega_{0}=2 \pi f_{0}$ with cross-sections $\sigma=(1-2) \pi R_{(\odot)}^{2}$. In models (Gordon 1967; Gordon, Liperovskii, \& Tsytovich 1971; Gordon 1973) Gordon explained widths of echo spectra with the help of scattering by microturbulance but as indicated by Gerasimova (Gerasimova 1979) the very high levels of Langmuir and ion-sound turbulance needed for reflections with crosssections $\sigma=10 \pi R_{\odot}^{2}$.For derivation of large cross-sections in the model (Wentzel 1981) some rigid constraints on solar magnetic fields are required. According to Chashei and Shishov (Chashei, \& Shisov 1994) scattering on large scale irregularities at heights $R=2 R_{\odot}$ in solar corona can provide an explanation of $10 \pi R_{\odot}^{2}$ cross sections. So until recently there were not understanding the main James' results.

In 1998 (Mel'nik 1998; Mel'nik 1999) the plasma theory of radar echo was suggested. It is development of the idea proposed by Gordon in 1967 about the important role of microturbulance in radar scattering. In this model radar signal 
is scattered by Langmuir and ion-sound turbulances that generated by Type III electrons. These electrons are said to have velocities $v_{0}=(0.3-0.6) c$ and propagate along open magnetic field lines in the solar corona. At each heights they generate Langmuir waves that are transformed into electromagnetic ones in the processes $l+i \rightleftharpoons t+i, l+l \rightleftharpoons t$ producing the fundamental and harmonic components of Type III bursts. The level of Langmuir turbulance estimated as $W=n^{\prime} m v_{0}^{2} / 12$ is enough high $W=\left(10^{-3}-10^{-4}\right) n T_{e}$. Another property of this turbulance is its anisotropy - wave vectors of Langmuir waves are directed along (primary waves) and against (secondary waves) direction of electron propagation into narrow cone with $\theta_{l}=10-20^{\circ}$. If electrons travel through regions with non-isothermal plasmas $T_{e}>T_{i}$ then Langmuir turbulance is the source of near isotropy ion-sound turbulance with the level $W_{s}=10^{-2} W_{l}$. Radar signal can be scattered by these Langmuir and ion-sound turbulances. Because Langmuir turbulance is anisotropy the scattering properties strongly depend on the relative orientations of propagation of high energy electron stream and radar signal. There are two cases.

If electrons propagate from the Sun to the Earth (in this case the active region is situated in the centre of the Sun) then wave vectors of Langmuir waves are parallel or antiparallel to wave vector of radar wave. The echo scattered in the processes $t+l \rightleftharpoons t+l$ travels backward to the Earth in the narrow cone $\pi \theta_{l}^{2}$. Scattering is most effective (the optical depth $\tau \geq 1$ ) at heights where Langmuir wave number equals radar wave number (Mel'nik 1999)

$$
k_{l}=k_{t} .
$$

As Langmuir waves are concentrated near wave number $k_{l}=\omega_{p e} / v_{0}$ then this equality is equivalent to

$$
\omega_{p e} / v_{0}=\omega_{0} / c
$$

Because of sharp directionality the scattering cross-section in this case is increased in comparison with that for isotropy scattering by a factor of $4 \pi / \pi \theta_{l}^{2}$ (Wentzel 1981) and equals $\sigma=4 S / \theta_{l}^{2}$, where $S$ is the visible section of Type III region. At heights $(1.3-1.6) R_{\odot} S \approx \pi R_{\odot}^{2}\left(R_{\odot}\right.$ is the solar radius) so one finds $\sigma \approx 100 \pi R_{\odot}^{2}$.

If electrons travel out of the Earth (active region is placed close to limb) the reflected signal coming on the Earth is weak (Mel'nik 1999), i.e. scattering cross section is small $\sigma<\pi R_{\odot}^{2}$.

When the radar signal reaches the heights where $\delta k_{l}=k_{t}\left(\delta k_{l}\right.$ is the width of Langmuir turbulance spectrum) scattering via processes $t+l \rightleftharpoons t+l$ is isotropic and $\sigma \approx \pi R_{\odot}^{2}$ in both cases of electron propagation (Mel'nik 1999).

Frequency shifts of radar echo is

$$
\triangle \omega=3 \omega_{0} \frac{\omega_{p e}}{\omega_{0}} \frac{v_{T e}^{2}}{v_{0}^{2}} \frac{\delta k_{l}}{k_{l}}
$$

and is determined by the electron temperature, the width of Langmuir turbulance and the electron velocity. For reasonable values of these parameters one can obtain observational frequency shifts from $20 \mathrm{kHz}$ to $60 \mathrm{kHz}$.

At the heights $\left(R>1.6 R_{\odot}\right)$ where $k_{l}<<k_{t}$ scattering due to processes $t+l \rightleftharpoons t+l$ is not effective (the optical depth $\tau<<1$ ) because of the small 
ratio $\left(\omega_{p e} / \omega_{0}\right)^{3}$. However at these heights scattering by ion-sound turbulance (processes $t+s \rightarrow t, t \rightarrow t+s$ ) is more efficient (the limiting height obtained from the condition $\tau=1$ for electron streams $n^{\prime} / n=10^{-5}$ equals $R \approx 5 R_{\odot}$ ). In this case the reflected signal consists of two symmetrical signals with respect to the frequency $\omega_{0}$ with

$$
\triangle \omega= \pm k_{s} v_{s}= \pm 2 \frac{\omega_{0}}{c}\left(\frac{T_{e}}{M}\right)^{1 / 2} .
$$

As the ion-sound turbulance is near isotropical the cross-section of scattering is defined only by the section $S_{s}$ of region with non-isothermal plasma $T_{e}>T_{i}$ and approximately equals $\sigma \approx S_{s}$. According to James' experiments reflections at great heights have different values $\Delta \omega$ and small cross-sections $\sigma<\pi R_{\odot}^{2}$, i.e. plasma consists of many regions with various electron temperatures. Because such reflections registered frequently we can conclude that the coronal plasma is irregular at these heights . The rarity of reflection at heights $R=5 R_{\odot}$ points to Type III electron stream has density $n^{\prime} / n \approx 10^{-6}$.

Thus our model allows to understand the main results of James' experiments. Besides his data give an opportunity to make conclusions about properties of the coronal plasma. Since the reflection heights, scattering cross-section and frequency shifts are determined by plasma parameters (density, temperature) and turbulance we can consider the radar experiments as a powerful method for diagnostic of the coronal plasma at the heights beyond the power of investigations by other methods. For this aim it is worthwhile using telescope with big effective areas such as UTR-2 $\left(1.5 \cdot 10^{5} \mathrm{~m}^{2}\right)$ and LOFAR $\left(10^{6} \mathrm{~m}^{2}\right)$.

This work was partially supported by INTAS, grant N96-0183.

\section{References}

Bass, F.G. and Braude, S.Ya. 1957 Ukr.Fiz.Zh., 11, 149

Chashei, I.V., and Shishov, V.I., 1994 Solar Phys. 149, 413

Gerasimova, N.N., 1979 Astron Zh., 56, 1303

Gordon, I.M., 1973 Space Science Rev., 15, 157

Gordon, I.M., 1967 Astron Tsir., N 447, 1

Gordon, I.M., Liperovskii, V.A., and Tsytovich, V.N., 1971 Astron Zh., 48, 70

James, J.C., 1966 ApJ, 146, 356

James, J.C., 1970 Mass. Inst., Center Space Research, Technic Report-70-2

Kerr, F.J. 1952 Proc. Inst. Radiophys. Electr., 40, 660

Mel'nik, V.N., 1998 Radiofizika i Radioastronomy, 3, 12;1998 Radiofizika i Radioastronomy, 3, 22

Mel'nik, V.N. 1999 Solar Phys.,184, 363

Mel'nik, V.N. 1999 Astronomy Letters, 25, 336

Wentzel, D.G., 1981 ApJ, 248, 1132 